\title{
Construction of cDNA library and preliminary analysis of expressed sequence tags from green microalga Ankistrodesmus convolutus Corda.
}

\begin{abstract}
Green microalga Ankistrodesmus convolutus Corda is a fast growing alga which produces appreciable amount of carotenoids and polyunsaturated fatty acids. To our knowledge, this is the first report on the construction of cDNA library and preliminary analysis of ESTs for this species. The titers of the primary and amplified cDNA libraries were $1.1 \times 10(6)$ and $6.0 \times 10(9) \mathrm{pfu} / \mathrm{ml}$ respectively. The percentage of recombinants was $97 \%$ in the primary library and a total of 337 out of 415 original cDNA clones selected randomly contained inserts ranging from 600 to $1,500 \mathrm{bps}$. A total of 201 individual ESTs with sizes ranging from 390 to 1,038 bps were then analyzed and the BLASTX score revealed that $35.8 \%$ of the sequences were classified as strong match, $38.3 \%$ as nominal and $25.9 \%$ as weak match. Among the ESTs with known putative function, $21.4 \%$ of them were found to be related to gene expression, $14.4 \%$ ESTs to photosynthesis, 10.9\% ESTs to metabolism, 5.5\% ESTs to miscellaneous, $2.0 \%$ to stress response, and the remaining $45.8 \%$ were classified as novel genes. Analysis of ESTs described in this paper can be an effective approach to isolate and characterize new genes from A. convolutus and thus the sequences obtained represented a significant contribution to the extensive database of sequences from green microalgae.
\end{abstract}

Keyword: Green microalgae; Ankistrodesmus convolutus; cDNA library construction; Expressed sequence tags. 